\title{
Direction of arrival estimation using a cluster of beams in a cone-shaped digital array radar
}

\author{
Micaela Contu, Marta Bucciarelli, Pierfrancesco Lombardo \\ DIET Dept. University of Rome "La Sapienza" \\ Rome, Italy \\ contu@die.uniroma1.it, marta.bucciarelli@uniroma1.it, \\ pierfrancesco.lombardo@uniroma1.it
}

\author{
Francesco Madia, Rossella Stallone, Marco Massardo \\ Seastema S.p.A. \\ Rome, Italy \\ Francesco.Madia@fincantieri.it, \\ rosella.stallone@seastema.it, marco.massardo@seastema.it
}

\begin{abstract}
In this paper some potential system and processing advantages of conformal cone shaped digital array radar have been investigated, in particular in relation to potential alternative approaches for angle estimation with respect to the traditional monopulse. First of all potential benefit in terms of reduction of the number of radiating elements is shown when a conical array is considered with respect to a traditional system formed by four planar arrays, if a coverage of $360^{\circ}$ must be assured. Secondly, having in mind an innovative digital array system where the received signals are analog to digital converted at element level and the corresponding data are possibly transferred to a central elaboration unit, an alternative approach is investigated for angular estimation. In this paper we derive the theoretical expression of the Cramer Rao Lower Bound for elevation angle estimation using a cluster of beams; we compare the limit performance of the traditional approach for angle estimation based on Sum and Difference beams with the approach based on a crowded cluster of $\mathrm{RX}$ beams properly spaced. The approaches show approximately equivalent performance, making the second particularly interesting for those situations where monopulse is known to experience performance degradation, as low elevation angle estimation; in this particular case an example of cluster design is shown, where the direct signal from a low altitude target must compete with a specular multipath.
\end{abstract}

Keywords-Digital array radar, conformal array, angle estimation

\section{INTRODUCTION}

It is well known that the basic characteristic on an array of radiating elements that makes it different with respect to traditional systems based on reflectors is the ability to form multiple beams with different characteristics and functionalities properly adjusting the phase or the delay of the signals received by each element in the array, [1].

In Phased Array Radars (PARs) this operation known as analog beamforming (ABF) is usually accomplished analogically through beamforming networks: in this case it is necessary to fix at design stage the number of the beams to be formed and their characteristics (beam steering direction, shape in terms of angular resolution and Peak to SideLobe Ratio PSLR, etc...). As an example, when a typical searching mode is considered, the use of a PAR must allow the simultaneous detection and accurate Direction of Arrival (DoA) estimation of a target in all the search space; the system must be designed to contemporarily form the appropriate sum and difference beams in each direction of interest for both applications, thus avoiding the necessity to re-irradiate to accurately estimate the targets' angle of arrival if detections occurred. As a consequence the beamforming network complexity increases with the number of directions to be monitored and the operative modes to be considered.

In Digital Array Radars (DARs) the analog to digital conversion at element or small sub-array level and the recording of all the amount of data for successive centralized processing allows completely changing the perspective with respect to operative modes based on analog beamforming, [1] [8]. As an example the coverage required in the searching mode is accomplished by foreseeing a wide transmitting beam (up to nearly omnidirectional in the "Ubiquitous Radar" theory) covered in reception by clusters of simultaneous beams at least in one angular direction. This is a first evident difference with respect to PARs using ABF, that search in all the desired directions using pencil beams both in transmission and reception allowing the execution of multiple functionalities using a limited number of complex beamforming network. Moreover the early digitization of the received signals allows reducing the required dynamic range and the requirements of isolation with respect to near clutter returns. It is apparent how all these advantages can be achieved at the expense of the increased computational load and data transfer rate to a central elaboration unit where beamforming, DoA estimation, adaptive nulling for interference cancellation, etc. take place. An ultimate analysis highlights that DAR provide an increased flexibility with respect to PAR allowing to develop multiple functions simultaneously and to implement alternative processing schemes increasing the efficiency of the exploitation of the acquired radar signals. For example, it could be possible to form only the Sum beams for detection in all the search space and then synthesize Difference beam for accurate DoA estimation only for the resolution cells when detection occurred. Moreover an alternative approach to angle of arrival estimation could be achieved using a crowded cluster of beams properly steered according to the direction where detection occurred.

In the present paper the considered and simulated digital array is cone-shaped. This particular shape factor is very interesting since, while in the elevation dimension the coverage is achieved with beam scanning operations, a $360^{\circ}$ azimuth coverage can be achieved using DBF without scanning but 
only circularly shifting the weight matrix around the beam steering direction; this provides potential benefits with respect to a more traditional architecture, where four planar arrays are used each one to cover an horizontal sector of $90^{\circ}$ with analogical or digital beam scanning. It has to be underlined that DBF not only allows a smart implementation of horizontal beamforming, but also provide an increased degree of technological feasibility to this conformal array, shifting the inherent criticalities from the analogic to the digital design, [8].

Having in mind an innovative array system like the one described, the aim of this paper is to investigate one of its numerous potentialities, comparing the performance of the traditional monopulse approach for DoA estimation, [9], possible both in a PAR and in a DAR architecture, and the approach feasible in a DAR based on a crowded cluster of RX beams properly spaced in an angular sector comprising the angular direction of the sum beam and for the resolution cell where a detection occurred. It is interesting to investigate this approach due to the fact that, as it is well known, [9], monopulse can suffer in particular situations where the investigated elevation angles are small and Sum and Difference beams can collect a great amount of land/sea clutter and multipath returns.

The paper is organized as follows. In section II the conical DAR system is described along with the reference operative scenario. In section III Cramer-Rao lower bound is derived for DoA estimation based on a cluster of beams and compared with the performance of the estimation based on Sum and Difference beams. An example of design for a cluster of beams to be employed for low altitude target DoA estimation is shown in IV. Finally we draw some conclusions in section V.

\section{SYSTEM DESCRIPTION AND POTENTIALITIES}

In the present paper the considered receiving $(\mathrm{RX})$ array is modelled as the "d.Radar", [10], which is being studied and demonstrated by Fincantieri S.p.A. and Seastema S.p.A. It is formed by a set of columns of radiating elements placed over the surface of a truncated cone as shown in Fig. 1 in a xyz reference system. Each element is connected to a receiver so that received signals are analog to digital converted at element level and it is supposed that the corresponding data are sent to a central elaboration unit. The array is designed in such a way that each horizontal section is represented by a circular array with the same number of elements with different inter-element distance as the height of the considered section decreases, while the vertical inter-element distance is constant and equal in each column. The overall structure has approximately a height of $0.8 \mathrm{~m}$ and a major diameter of 1.42 meters.

As hinted in the introduction a cone-shaped structure supplied with DBF to provide $360^{\circ}$ coverage can present advantages due to this conformal shape factor with respect to a traditional four-planar faces array, Fig. 2. Indeed in this case beamforming in every horizontal direction can be achieved by circularly shifting the weight matrix according to the beam steering direction that is by elaborating in the same way the signals digitized at element level received from a properly selected active sector of the array; in that way a beam with the same desired characteristics of width $\delta \phi$ is achieved in every direction without beam scanning (Fig. 2a). On the other hand when four planar arrays are used each one to cover an horizontal sector of $90^{\circ}$ with analogical or digital beam scanning, degraded patterns are achieved off-boresight with maximum width equal to $\delta \phi_{\max }$ (Fig. 2b). Matching the approximate expressions of the beamwidth, in the hypothesis of equal element spacing, the ratio of the perimeters of both horizontal sections can be evaluated as a quality parameter showing the ratio between the number of elements required in the circular array with respect to the square array

$$
\Delta N_{e l}=\pi \sin \left(\phi_{0}\right) / 4 \cos \left(\alpha_{0} / 2\right)
$$

For sectors of active elements wider than $120^{\circ}$, the proposed coarse dimensioning suggests that using a conical array allows sparing radiating elements, mainly when the performance of the different configurations of the arrays are compared in the worst case, that is when the maximum beamwidth $\delta \theta_{\max }$ is taken into account.

One of the typical operative conditions of a radar is represented by the "searching mode". In the considered system it is supposed that a transmitting (TX) antenna radiates with a beam which is narrow in the azimuth dimension and wide in elevation; therefore, using the conceived RX array with a properly dimensioned active sector and tapering functions specifically tailored to cope with a 3D displacement of the elements of the array, multiple Sum beams displaced in the vertical dimension must be formed to cover the nominal illuminated angular aperture along with the corresponding Difference beams; Fig. 4 shows the case of a TX aperture in elevation nearly equal to $8^{\circ}$ covered by $3 \mathrm{RX}$ beams for different steering directions of the central beam, in particular $15^{\circ}$ in elevation and $0^{\circ}$ in azimuth in Fig. $4 \mathrm{a}$ and $5^{\circ}$ in elevation and $0^{\circ}$ in azimuth in Fig. 4b. From this second case it is apparent how lower Sum and expecially Difference beams in the cluster may suffer from clutter and multipath arising from low-height target. This may suggest the use of a different approach for DoA estimation, in particular for the elevation angle, which is not based on Difference beams as the monopulse. Indeed when detection occurs in a certain beam and at a certain resolution cell, if data acquired by all elements are available at a central elaboration unit before beamforming, accurate elevation angle estimation can be be based on the exploitation of a crowded clusters of sum beams properly displaced in the angular area of interest. In the following section the feasibility of this approach is investigated.

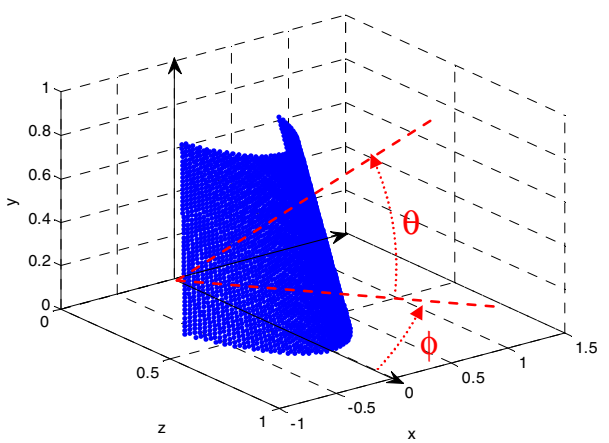

Fig. 1. Active sector $\left(120^{\circ}\right)$ of the considered cone-shaped array radar 


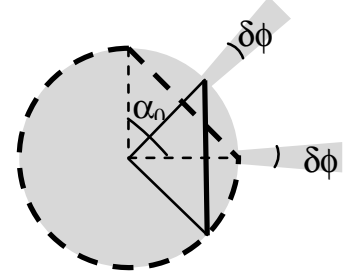

(a)

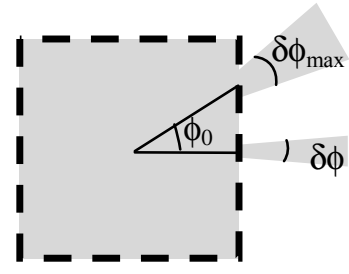

(b)
Fig. 2. Horizontal sections of (a) the cone and (b) the 4-planar faces arrays.

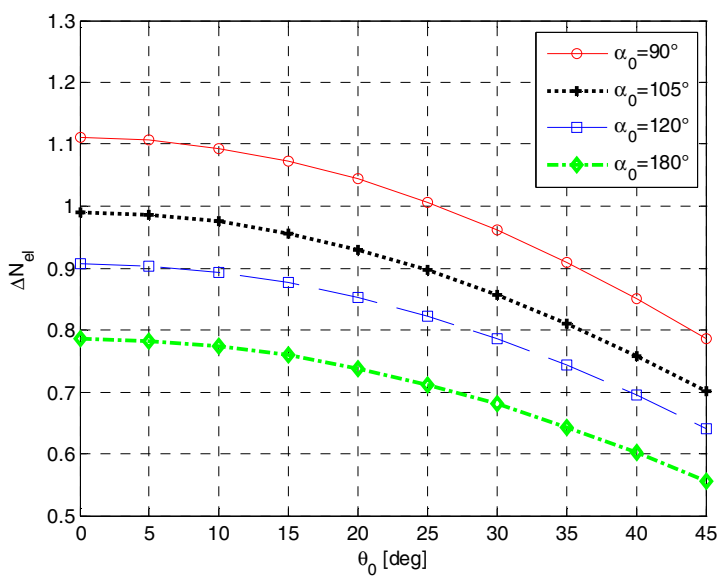

Fig. 3. Ratio between the number of elements required in circular DAR with respect to a square array as a function of the steering direction $\theta_{0}$ for different widths of the active section $\alpha_{0}$.
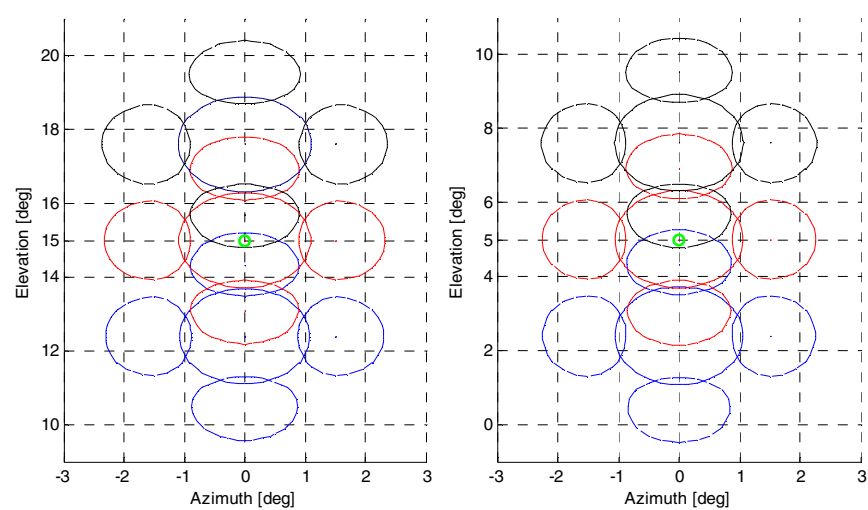

Fig. 4. Sum and Difference beam apertures for a central steering of (a) $15^{\circ}$ in elevation ad $0^{\circ}$ in azimuth and (b) $5^{\circ}$ in elevation and $0^{\circ}$ in azimuth.

\section{CRAMER-RAO LOWER BOUND DERIVATION FOR DOA ESTIMATION BASED ON A CLUSTER OF BEAMS}

In this paragraph the expression of the Cramer Rao Lower Bound (CRLB) is derived for the elevation DoA estimation using a cluster of beams. For this reason in the following the azimuth direction of the signal will be considered known and fixed to $\phi_{0}=0$.

The received signal $\mathbf{x}$ is modelled as

$$
\mathbf{x}(\theta)=A_{0} G_{T X}\left(\theta, \theta_{0}\right) G_{E}(\theta) \mathbf{T s}(\theta)+\mathbf{T n}
$$

$A_{0}$ is an amplitude factor depending on the signal path and on the radar equation, $G_{T X}\left(\theta, \theta_{0}\right)$ is the gain at the direction $\theta$ of the transmitting pattern steered towards $\theta_{0}, G_{E}(\theta)$ represents the gain provided by each antenna element to a signal $\mathbf{s}(\theta)$ coming from the direction $\theta, \mathbf{T}$ is the transformation matrix that allows the switch from an element-wise to a cluster based representation and $\mathbf{n}$ is the vector accounting for noise samples with null expected value and variance equal to $\sigma_{n}$. $\mathbf{s}(\theta)$ is defined as

$$
\mathbf{s}(\theta)=\left[\begin{array}{c}
\exp \left(-j 2 \pi / \lambda\left\langle\mathbf{p}_{\mathbf{1}}, \mathbf{u}(\theta)\right\rangle\right) \\
\vdots \\
\exp \left(-j 2 \pi / \lambda\left\langle\mathbf{p}_{\mathbf{N}}, \mathbf{u}(\theta)\right\rangle\right)
\end{array}\right]
$$

where $\lambda$ is the wavelength, $\mathbf{p}_{\mathbf{i}}$ is the vector containing the xyz coordinates of the ith element in the array for $i=1, \ldots, N$, $\mathbf{u}(\theta)$ is the direction cosine vector and the symbol $\langle\cdot$, accounts for the inner product.

The transformation matrix $\mathbf{T}$ can be written as

$$
\mathbf{T}=\left[\begin{array}{c}
\mathbf{W}_{\mathbf{1}} \mathbf{s}^{*}\left(\theta_{1}\right) \\
\vdots \\
\mathbf{W}_{\mathbf{K}} \mathbf{s}^{*}\left(\theta_{K}\right)
\end{array}\right]
$$

where $\mathrm{K}$ is the number of beams in the cluster, symbol * refers to the complex conjugate operation and $\mathbf{W}_{\mathbf{k}}$ is the diagonal matrix accounting for amplitude tapering.

The probability density function of $\mathbf{x}(\theta)$ can be modelled as Gaussian and equal to

$$
f[\mathbf{x}]=\frac{1}{2 \pi \cdot \operatorname{det}(M)} \exp \left\{-\left[\mathbf{x}-A_{0} \mathbf{v}\right]^{H} \mathbf{M}^{-1}\left[\mathbf{x}-A_{0} \mathbf{v}\right]\right\}
$$

where $\mathbf{v}$ and $\mathbf{M}$ are the expected value of $\mathbf{x}$ and the covariance matrix of the disturbance

$$
\mathbf{v}(\theta)=G_{T X}\left(\theta, \theta_{0}\right) G_{E}(\theta) \mathbf{T s}(\theta)
$$

$$
\mathbf{M}=\sigma_{n}^{2} \mathbf{T T}^{H}
$$

In the previous expression symbol $\mathrm{H}$ has been used to indicate complex conjugate and transpose operation, while $\operatorname{det}(M)$ referred to the determinant of matrix $\mathbf{M}$.

The unknowns in (5) are the real and imaginary part of $A_{0}$ and the elevation direction $\theta$. Therefore the Fisher information matrix can be written as

$$
\mathbf{J}=-E\left\{\left[\frac{\partial}{\partial \boldsymbol{\eta}} \ln (f(\mathbf{x} / \boldsymbol{\eta}))\right]^{T}\left[\frac{\partial}{\partial \boldsymbol{\eta}} \ln (f(\mathbf{x} / \boldsymbol{\eta}))\right]\right\}
$$


where $\eta=\left[A_{O R} A_{0 I} \theta\right]$ and apex $\mathrm{T}$ accounts for the transpose operation. The third element on the diagonal of the inverse of the matrix $\mathbf{J}$ is the searched accuracy, equal to

$$
\sigma_{\theta}^{2}=\mathbf{J}^{-1}(3,3)=2 \frac{\left|A_{0}\right|^{2}}{\sigma_{n}^{2}} \dot{\mathbf{v}}^{H}\left[\mathbf{M}^{-1}-\frac{\mathbf{M}^{-1} \mathbf{v}{ }^{H} \mathbf{M}^{-1}}{\mathbf{v}^{H} \mathbf{M}^{-1} \mathbf{v}}\right] \dot{\mathbf{v}}
$$

where $\left|A_{0}\right|^{2} / \sigma_{n}^{2}$ is the signal to noise ratio at element level $\left(\mathrm{SNR}_{\mathrm{el}}\right)$ and $\dot{\mathbf{v}}$ is the first derivative of the vector $\mathbf{v}$ with respect to the direction $\theta$.

Fig. 5 shows the standard deviation of the estimation of $\theta$ normalized with respect to the nominal beamwidth of a RX pattern in the elevation dimension as a function of the difference of the DoA $\theta$ and the steering direction of the central beam in the cluster, $\theta_{T}=5^{\circ}$ in this case. All the sum beams have been formed considering properly modified Taylor windows both in azimuth and elevation with a PSLR of $42 \mathrm{~dB}$ and $36 \mathrm{~dB}$ respectively. The Difference beams in elevation are built using a Bayliss tapering with the same parameters. The signal to noise ratio at element level $\mathrm{SNR}_{\mathrm{el}}$ has been set equal to $-20 \mathrm{~dB}$, therefore a peak SNR on the central beam of nearly $13 \mathrm{~dB}$ is achieved: the gain of nearly $33 \mathrm{~dB}$ is provided by the coherent summation of the amplitude weighted signals received by nearly 3500 elements in the considered active sector.

Two different cases are considered: in the first case (Fig. 5a) we supposed an omnidirectional TX pattern, while in the second case (Fig. 5b) a directive transmission pattern has been considered with a $-3 \mathrm{~dB}$ aperture in elevation $\mathrm{B}_{\mathrm{TX} \text {-el }}=8^{\circ}$ nearly equal to three times the beamwidth of the generic RX pattern $\mathrm{B}_{\mathrm{RX} \text {-el }}$. In both cases omnidirectional elements have been considered and an active sector of $120^{\circ}$ has been used for beamforming. Four curves are shown. The green one is related to the CRLB achieved considering a cluster formed by 3 Sum beams $(3 \Sigma)$ and 3 Difference beams $(3 \Delta)$ thus representing the lower bound for the monopulse accuracy in the covered area. The other curves refer to the cases of cluster of $\mathrm{K}$ uniformly spaced beams $(K=5,7,13)$ covering the same elevation extent as the $3 \Sigma$ case, that is $\left[\theta_{\mathrm{T}}-\mathrm{B}_{\mathrm{TX}-\mathrm{el}} / 2, \theta_{\mathrm{T}}+\mathrm{B}_{\mathrm{TX}-\mathrm{el}} / 2\right]$. This means that as the number of beams in the cluster increases, the angular separation between beam steering directions decreases.

From Fig. 5 it is apparent how the limit performance of the $3 \Sigma+3 \Delta$ configuration is equivalent to the limit performance of the DoA estimation based on cluster of crowded Sum beams, especially in the interval $\left[-4^{\circ}, 4^{\circ}\right]$; as the number of beams increases, ripple's amplitude decreases since the coverage becomes uniform in the area of interest and the performance is less affected by pattern peaks with null derivative. In Fig. 5a it is apparent how a slight slope appears evidently as the number of beams increases; this effect arise from the asymmetry of the distribution of elements over the considered conical array surface, where higher elements are closer to each other than lower elements. In Fig. $5 b$ the presence of a directive pattern in transmission is evident and shows itself as a tapering of the accuracy mainly due to the gain provided by the TX beam in each considered direction.

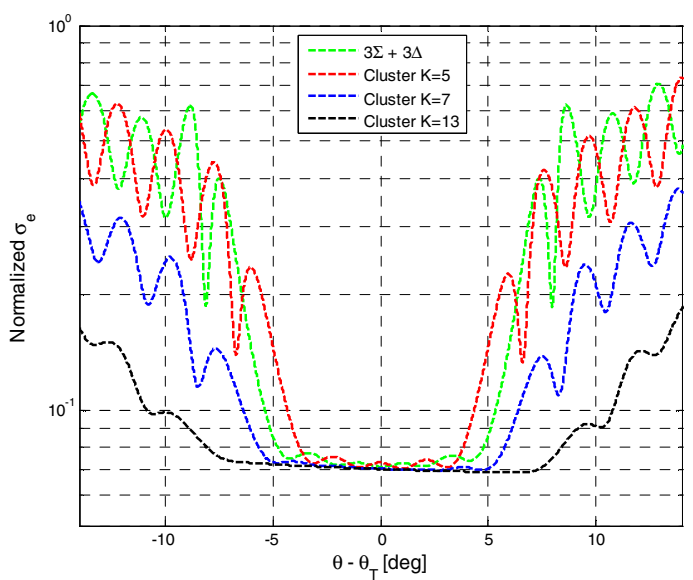

(a)

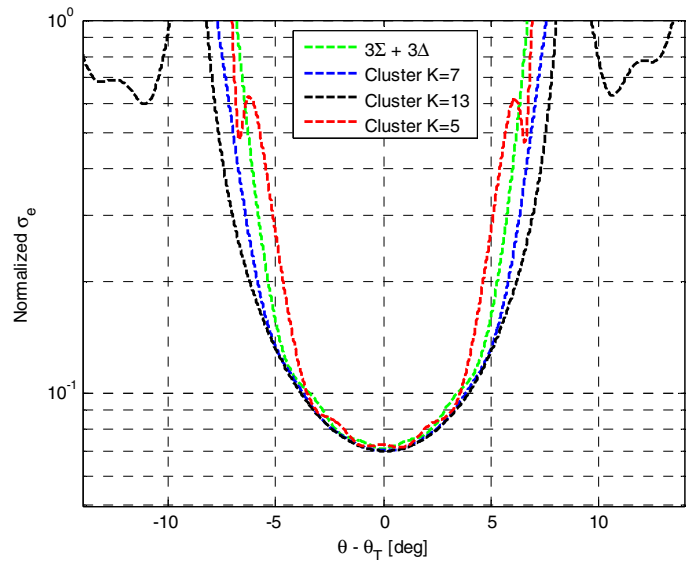

(b)

Fig. 5. Normalized Cramer Rao Lower Bound for the accuracy of the estimation od the DoA $\theta$ (a) with omnidiractional pattern in TX and (b) for a directive pattern in TX.

Ultimately the derivation of the CRLB for the accuracy of elevation DoA estimation using a cluster of beams allowed us to compare the limit performance of an estimation approach which uses only Sum beams and the traditional approach using Sum and Difference beams and to assess an actual equivalence on the approaches. The main difference lies in the fact that although in both approaches all the Sum beams have positive tilts with respect to the horizon, Difference beams at very low elevation can heavily affect the estimation performance when significant land or sea clutter and multipath signals occur. Nevertheless also in the case of DoA estimation performed using a crowded cluster of Sum beams the design of the cluster needs to be accurate and to take into account the operative scenario, especially when target detection occurs at very low altitudes.

\section{EXAMPLE OF CLUSTER DESIGN}

In this section an example of design of the cluster of Sum beams to be employed in DoA estimation is provided. It is based on an operative scenario where signals from a low altitude target are received through a direct and a specular reflected path in the flat earth model, Fig. 6. 
The steering direction of the lower beam in the cluster is selected to provide a gain of at least $3 \mathrm{~dB}$ to the direct signal from direction $\theta$ with respect to the multipath signal received from the direction $\theta_{M}$ in the worst condition of operation foreseen for the radar that is the minimum target height to take into account, experiencing the corresponding gain loss at the target direction $\theta$. The steering direction of the remaining beams in the cluster can be selected to uniformly cover the desired angular aperture. Assuming a specular reflection of the multipath signal, a distance $\mathrm{D}=20 \mathrm{~km}$ and an antenna height of $15 \mathrm{~m}$, as expected Fig. 7 shows how the design becomes more demanding as the target height decreases with a loss of $-17 \mathrm{~dB}$ in the target direction for $H_{t g}=30 \mathrm{~m}$ (Fig. 7a) and $-7 \mathrm{~dB}$ for $H_{\text {tgt }}=60 \mathrm{~m}$ (Fig. 7b). In the previous example an omnidirectional TX pattern is taken into account; a further slight loss is expected when a directive pattern in employed in transmission.

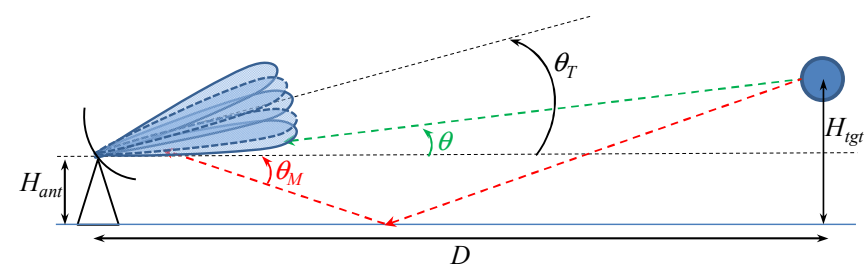

Fig. 6. Operative scenario

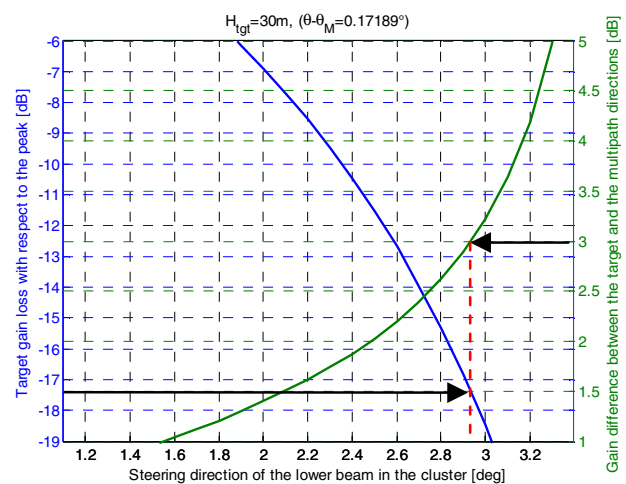

(a)

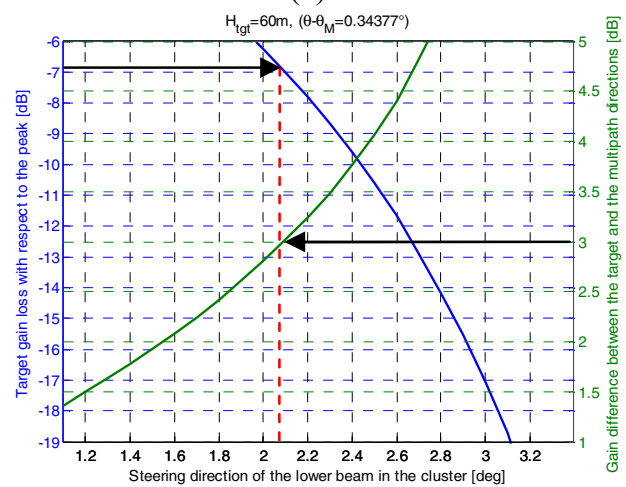

(b)

Fig. 7. Gain loss in the target direction (blue curve) and gain difference in the target and multipath directions (green curve) for a target height (a) of $30 \mathrm{~m}$ and (b) of $60 \mathrm{~m}$

\section{CONCLUSIONS}

Some potential system and processing advantages of conformal conical DAR have been investigated, particularly in defining alternative approaches for DoA estimation.

A coarse dimensioning based on the matching of the pattern beamwidth in the azimuth dimension showed that the conical shape factor allows to guarantee without horizontal beam scanning $360^{\circ}$ coverage using less elements with respect to the case of four planar arrays, each assuring a coverage of $90^{\circ}$ with beam scanning. This result appeared to be valid as the sector of active elements used for beamfoming in the conical array case increases for all the possible steering directions.

Having in mind an innovative digital array system like the one described, we derived the theoretical expression of the CRLB for elevation angle estimation using a cluster of beams and we compared the limit performance of the traditional approach based on sum and difference beams, possible both in a PAR and in a DAR architecture, and the approach feasible in a DAR based on a cluster of RX beams properly spaced according to the direction and for the resolution cell where a detection occurred. The approaches proved to be approximately equivalent, making the second particularly interesting for those situations where monopulse is known to experience performance degradation, as low elevation angle estimation. In this case an example of the design of the cluster of beams in presence of specular multipath has been provided

\section{ACKNOWLEDGMENT}

The Authors thank Roberto Ronconi from Seastema S.p.A. for the useful discussion about the "d.Radar" system.

\section{REFERENCES}

[1] M. I. Skolnik, "Phased Array Radar Antennas," in Radar Handbook, 3rd ed., McGraw-Hill Professional, 2008.

[2] W. L. Melvin, J. A. Scheer, "Array Processing and Interference Mitigation techniques," in Principles of Modern radar, Advanced Techniques, SciTech Publishing, 2012.

[3] M. I. Skolnik, "Opportunities in radar-2002," Electronics \& Communication Engineering Journal, vol. 14, no. 6, pp. 263-272, 2002.

[4] C. Tarran, "Advances in affordable Digital Array Radar," 2008 IET Waveforme Diversity and Digital Radar Conference, 8-9th December 2008, London, UK.

[5] F. Le Chevalier, "Future concepts for electromagnetic detection from space-time-frequency resources management to wideband radars", IEEE Aerosp. Electron. Syst. Mag., vol. 14, no. 10, pp. 9-17, 1999.

[6] D. J. Rabideau, P.Parker, "Ubiquitous MIMO multifunction digital array radar", $37^{\text {th }}$ Asilomar Conference on Signals, Systems and Computers, 9-12 Nov. 2003, Pacific Grove, CA, USA.

[7] B. Cantrell, J. de Graaf, F. Willwerth, G. Meurer; L. Leibowitz, C Parris; R.Stapleton, "Development of a Digital Array Radar (DAR)", IEEE Aerosp. Electron. Syst. Mag., vol. 17, no. 3, pp. 22-27, 2002.

[8] J.H. Hunter, T.W.Miller, "Digital beamforming demonstration radar", Antennas and Propagation Society International symposium, 7-11 May 1990, Dallas, Tx, USA.

[9] S. M. Sherman, D. K. Barton, "Monopulse Principles and Techniques", Artech House, 2011.

[10] F. Madia, A. Maestrini, "Radar bistatico", pending Italian patent N. RM2014A000005, Jan 9, 2014. 